\title{
Survivin expression in in situ and invasive breast cancer relates to COX-2 expression and DCIS recurrence
}

\author{
N Barnes', P Haywood', P Flint', WF Knox ${ }^{2}$ and NJ Bundred*,I \\ 'Department of Academic Surgery, South Manchester University Hospital, Manchester, UK; ${ }^{2}$ Department of Pathology, South Manchester University \\ Hospital, Manchester, UK
}

In lung cancer cyclooxygenase-2 (COX-2) expression has been reported to stabilise survivin, an inhibitor of apoptosis (IAP) which prevents cell death by blocking activated caspases. COX-2 expression limits the ubiquitination of survivin, protecting it from degradation. To determine if COX-2 expression in breast cancer showed an association with survivin expression, we assessed the levels of each protein in ductal carcinoma in situ (DCIS) and invasive breast cancer (IBC); relating expression patterns to recurrence of DCIS after surgery. Patterns of COX-2 and survivin expression were determined by intensity-graded immunohistochemistry of the primary tumours. Patients with DCIS $(n=161)$ which had either recurred $(n=47)$ or shown no evidence of recurrence $(n=1 \mid 4) 5$ years following primary surgery were studied. These were compared to 58 cases of IBC. Survivin was expressed in the cytoplasm of $59 \%$ of DCIS and 17\% of IBC. High levels of both cytoplasmic survivin and COX-2 expression significantly correlated to DCIS recurrence. COX-2 expression was present in $72 \%$ of DCIS, and levels of expression positively correlated with cytoplasmic survivin expression in DCIS and invasive disease. The majority of DCIS that recurred expressed both proteins (69\%) vs 39\% nonrecurrent. Recurrence was not seen in DCIS lacking both proteins at 5 years $(P=0.00 \mathrm{I})$. Expression of the IAP survivin is increased in DCIS and correlates closely with COX-2 expression. Increased expression of IAP, (leading to reduced apoptosis) may explain the effect of COX-2 in increasing recurrence of DCIS after surgical treatment.

British Journal of Cancer (2006) 94, 253-258. doi:I0.1038/sj.bjc.6602932 www.bjcancer.com

Published online 17 January 2006

(c) 2006 Cancer Research UK

Keywords: cyclooxygenase; apoptosis; survivin; breast; DCIS; recurrence

The incidence of the preinvasive breast cancer ductal carcinoma in situ (DCIS) has increased by over five-fold since the introduction of national screening programs (Ernster et al, 2000). The recurrence rate in the NSABP B-17 trial at 10 years following breast conserving surgery and radiotherapy remains at almost $12 \%$ (Fisher et al, 1986). There is, therefore, a need to identify novel predictors of recurrence risk and potential targets for therapy. Cyclooxygenase-2 (COX-2) is an inducible rate-limiting enzyme in the conversion of arachidonic acid to prostaglandins. It is induced by a number of promoters including c-erbB2/(HER2) and NF $\kappa \beta$ (Smith et al, 2000). We have previously shown that COX-2 expression is related to a poor prognostic phenotype of DCIS (Boland et al, 2004), but there are no published data on whether COX-2 expression affects recurrence of DCIS. Survivin is a member of the inhibitors of apoptosis protein (IAP) family, which includes proteins such as X-IAP, H-IAP and survivin (Ambrosini et al, 1997; Schimmer, 2004), that have effects on both cell cycle regulation and apoptosis. The antiapoptotic properties of the IAP's lie in their ability to block the activation of caspases (mainly 3, 7 and 9) in the cell cytoplasm, which are key regulators of classical

*Correspondence: Professor NJ Bundred, Department of Academic Surgery, Research and Education Building 2nd Floor, South Manchester University Hospital, Southmoor Road, Wythenshawe, Manchester, M23 9LT, United Kingdom; E-mail: bundredn@man.ac.uk

Received 10 August 2005; revised 16 November 2005; accepted 29 November 2005; published online 17 January 2006 apoptosis (Schimmer, 2004). In addition to their antiapoptotic effects, IAP's have been shown to regulate cell cycle progression and cell signalling (Schimmer, 2004). Survivin is expressed in fetal tissues but is absent from normal terminally differentiated cells (Ambrosini et al, 1997) (except the basal colon epithelial cells where it may act to limit the stem cell population (Zhang et al, 2001)). It is, however, overexpressed in a wide range of human invasive cancers including breast (Tanaka et al, 2000), colon (Kawasaki et al, 1998), gastric (Lu et al, 1998), endometrial (Saitoh et al, 1999) and lung (Monzo et al, 1999). COX-2 overexpression has been shown to stabilise survivin in nonsmall cell lung cancer by preventing its ubiquitination (Krysan et al, 2004) leading to the decreased rates of apoptosis seen in COX-2 overexpressing lung tumours.

The aim of the study was to determine the correlation between COX-2 and survivin expression in DCIS and their relationship to expression levels in invasive disease and recurrence after surgery.

\section{MATERIAL AND METHODS}

\section{Immunohistochemistry}

Archival blocks of formalin-fixed paraffin-embedded tissue were selected, following confirmation that the blocks contained either pure DCIS or invasive breast cancer (IBC) by an experienced breast pathologist (WFK). Staining for estrogen receptor (ER), HER2 and Ki67 and COX-2 have been previously described 
(Boland et al, 2004). For survivin, the slides were dewaxed in xylene and rehydrated through graded alcohols. Following immersion in $0.2 \% \mathrm{H}_{2} \mathrm{O}_{2}$ in methanol for $10 \mathrm{~min}$, the slides were placed in citrate buffer for antigen retrieval under pressure. Nonspecific binding was blocked with $10 \%$ normal goat serum in phosphate-buffered saline (PBS), with avidin. The primary antisurvivin antibody (Ab469 AbCam, Cambridge UK) was diluted $1: 400$ in normal goat serum/PBS with biotin and incubated on the slides at $4{ }^{\circ} \mathrm{C}$ overnight. Following further washes in PBS, the biotinylated goat anti-rabbit antibody secondary (BA-1000, Vector, UK) was applied for $1 \mathrm{~h}$. After washing in PBS, the sections were incubated in $\mathrm{ABC}$ Vectastain elite reagent (Vector, UK) for $35 \mathrm{~min}$. Staining was visualised with DAB, slides counterstained with $20 \%$ haematoxylin, dehydrated, cleared and mounted.

\section{Evaluation of immunostaining}

Staining was assessed by light microscopy without the knowledge of clinico-pathological features or patient outcome. For ER and Ki67 the percentages of positively staining nuclei were determined. At least 1000 cells were counted for each case, at $\times 400$ magnification. ER status was taken to be positive if $\geqslant 5 \%$ of cells were labelled. The membranous and cytoplasmic staining of HER2 was assessed semiquantitatively using the proportion of positively staining cells and degree of staining intensity compared to adjacent normal tissue; samples scored 0 to $3+$ as previously described (Boland et al, 2004). The cytoplasmic staining of COX-2 was scored 0 (absent) to $3+$ (strong) based on the extent and intensity of staining as previously described (Boland et al, 2004), COX-2 positivity was defined as a score of $\geqslant 2$. Survivin staining was scored for both cytoplasmic and nuclear staining independently. The AbCam ab469 antibody used has been shown to detect all three splice variants of survivin (Fortugno et al, 2002) which together are present in both the cytoplasm and the nucleus. Cytoplasmic staining was scored 0 (absent) to $3+$ (strong); scores of $\geqslant 2$ taken as positive. When cells displayed nuclear survivin the proportion of positive cells out of at least 1000 was determined (Figure 1).

\section{Patient selection}

Women diagnosed with pure DCIS $(n=161)$ between 1979 and 1999 were selected, of which, 47 had recurred within 5 years

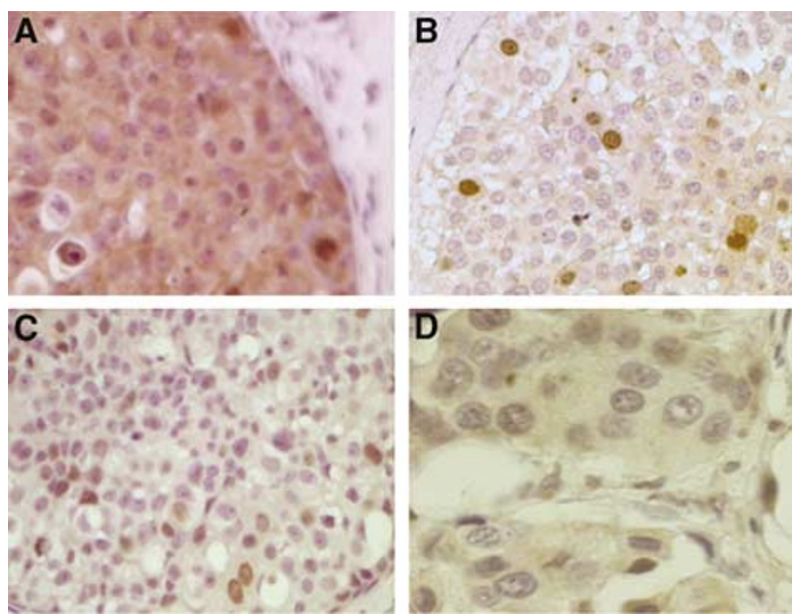

Figure I Immunohistochemical staining for surviving. (A) Cytoplasmic survivin positive DCIS 3+; (B) Cytoplasmic survivin positive DCIS 2+; (C) Nuclear survivin alone in DCIS. (D) IBC showing cytoplasmic survivin $1+$. (median time to recurrence 21 months; range 10-60) and 114 were recurrence free after 5 years of follow-up. In all, 13 of the recurrences were invasive disease and 34 were further DCIS. Breast conserving surgery had been performed in 60 of the nonrecurrent and 43 of the recurrent DCIS, and mastectomy in 39 nonrecurrent and eight of the recurrent cases. In our department women with a DCIS exceeding $4 \mathrm{~cm}$ would be considered for mastectomy rather than breast conserving surgery. Current policy is to ensure that all wide local excision samples have at least $1 \mathrm{~mm}$ clear surgical excision margins. However, this data set used archival specimens, a subset of which did not have clear margins (as they were treated before the benefit of clear margins was proven) therefore, margin status was included in the multivariate analysis of the results. There were not sufficient recurrences following mastectomy to identify significant differences in the nature of recurrence between the mastectomy and breast conserving surgery groups. A random selection of patients with IBC who had undergone surgery between 1980 and 1999 were selected from a tumour bank in our department to compare COX-2 and survivin expression in invasive disease. It was ensured that the invasive samples represented all tumour grades in similar proportions to the DCIS sample. Tumour nuclear grade was determined following review of the original pathology report. Ethical approval was given by the South Manchester University Hospital Ethics Board.

\section{Statistical analysis}

The relationship between receptor coexpression tumour size, nuclear grade, ER status and recurrence were assessed by the $\chi^{2}$ test. The relationship between Ki67 and receptor expression was assessed using the Kruskal-Wallis test. Correlation coefficients were generated using Spearman's nonparametric correlation. Kaplan-Meier survival plots were generated with log-rank significance. The multivariate analysis was performed using the COX proportional hazards analysis. Significance tests were twotailed and 5\% significance level was used throughout. Analysis was carried out using SPSS 10.0 for windows.

\section{RESULTS}

\section{Survivin expression in DCIS}

In total 102 cases of DCIS were stained for survivin; 69 with no evidence of recurrence at 5 years and 33 with recurrence by 5 years of follow-up. The remaining blocks had insufficient tissue left as COX-2, ER, Ki67 and HER2 staining had already been performed. Survivin was present in both the cytoplasmic and nuclear subcellular compartments. In all, 32 cases (31\%) showed no survivin staining (nuclear or cytoplasmic), 10 of $102(10 \%)$ showed nuclear staining alone, 30 of $102(29 \%)$ showed both cytoplasmic and nuclear staining and 30 of $102(29 \%)$ showed cytoplasmic staining alone. The DCIS that recurred was significantly more likely to express moderate to strong $(2$ to $3+)$ levels of cytoplasmic survivin (76\%) compared to $37 \%$ of nonrecurrent disease $(P=0.0001)$ (Table 1). Cytoplasmic survivin expression score correlated with COX-2 expression score (Spearman's correlation coefficient $0.322, P=0.001$ ). None of the 10 cases that showed nuclear survivin alone recurred within 5 years (Table 1).

There were no significant associations between cytoplasmic survivin expression and the clinico-pathological factors of age, nuclear grade, pathological tumour size, ER, HER2, HER4 status or proliferation (Ki67) (data not shown).

\section{COX-2 expression in DCIS}

As we have previously demonstrated (Boland et al, 2004), COX-2 was highly expressed in the cytoplasm of DCIS cells. Overall, 116 of the 161 cases studied (72\%) were COX-2 positive (scores of 
Table I COX-2 and survivin expression by tumor type

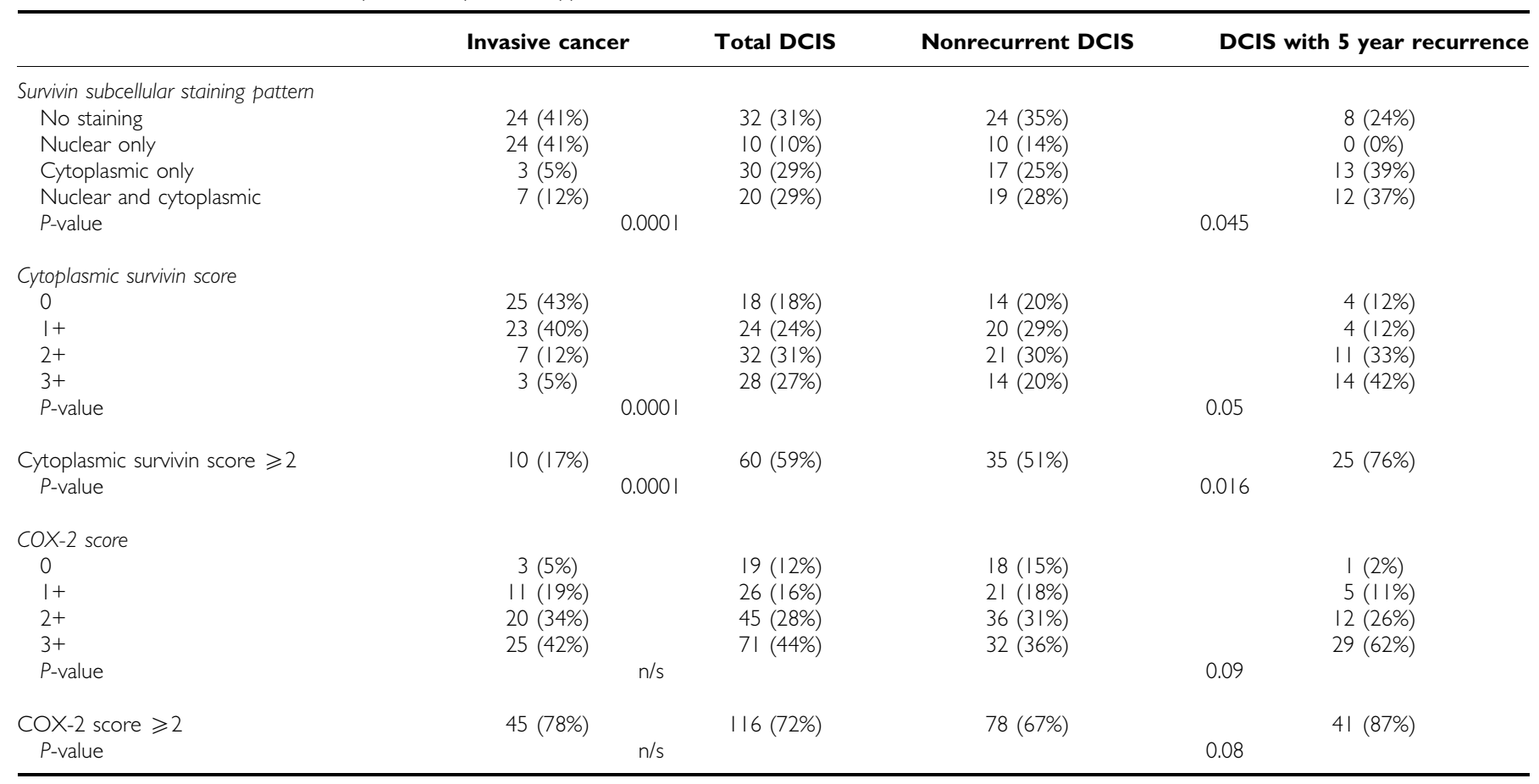

NOTE: $\mathrm{n} / \mathrm{s}=$ not statistically significant.

moderate or strong staining). Greater COX-2 expression was seen in DCIS that recurred than the nonrecurrent cases $(P=0.010$; Table 1). The majority of DCIS that recurred expressed moderate or strong COX-2 (Table 1). Only one of the 47 cases that recurred expressed no COX-2 (2\%) and only five (11\%) expressed weak COX-2 (Table 1), compared to $16 \%(18 / 114)$ and $18 \%(21 / 114)$ cases of nonrecurrent DCIS expressing none or weak staining, respectively. We have previously demonstrated that COX-2 expression correlated with the Type 1 Tyrosine kinase receptor c-erbB2/HER2 expression (Boland et al, 2004) and this association was upheld in this new series of women (Spearman's correlation coefficient $0.271, P=0.001$ ).

\section{Differential survivin staining in DCIS $v s$ IBC}

To assess the differences between survivin expression between DCIS and IBC a panel of 58 IBC's were stained for survivin and COX-2. The grade variation of the invasive cancer was chosen to be similar to that of the DCIS, with six (10.7\%) of the invasive cases being grade $1,13(23.2 \%)$ grade 2 and $37(66.1 \%)$ grade 3. Overall, survivin was expressed at moderate to strong levels in $10 / 58(17 \%)$ of IBC, compared to 60 of $102(60 \%)$ of patients with DCIS $(P=0.0001$; Table 1$)$. IBC showed weak staining in a further 23 of $58(40 \%)$ of cases. The distribution of nuclear and cytoplasmic survivin also differed significantly between IBC and DCIS, with a higher proportion of invasive cases showing nuclear staining alone 24 of $58(41 \%)$, compared to 10 of 102 $(10 \%)$ cases of DCIS. Fewer cases of IBC showed evidence of cytoplasmic survivin expression, both alone (12 vs 30\%), or with coexisting nuclear survivin (5 vs 29\%) (Table 1). There was no difference in COX-2 expression, in this dataset, between DCIS and IBC.

The presence of cytoplasmic survivin correlated with COX-2 expression in IBC (correlation coefficient $0.28, P=0.004$ ) as it did in DCIS. No IBC expressed survivin without COX-2.

\section{Cytoplasmic survivin and COX-2 coexpression relates to DCIS recurrence}

Overall, 13 (17\%) cases of DCIS expressed neither protein, 29 (28\%) expressed COX-2 alone, nine (8\%) expressed cytoplasmic survivin alone and $51(50 \%)$ expressed both proteins.

Of the cases that recurred $70 \%(n=23)$ expressed both COX-2 and cytoplasmic survivin. In contrast, none of the cases that did not express either protein $(n=13)$ recurred within 5 years $(P=0.013)$ (Table 2). Coexpression patterns did not relate to cell proliferation, tumour nuclear-grade, ER or HER2 status (Table 2). The relationship between COX-2, survivin and DCIS recurrence was upheld when looking only at the cases that had undergone breast conserving surgery $(P=0.021)$ (Table 2).

When the 5-year cumulative disease free survival was plotted against cytoplasmic survivin status as Kaplan-Meier graphs, DCIS that was cytoplasmic survivin positive (scores of 2 and $3+$ ) had a significantly poorer 5-year outcome than cytoplasmic survivin negative cases (scores of 0 and $1+$ ) (Figure 2A and B). The number of DCIS cases recurring by 5 years also increased with increasing COX-2 score (Figure 2C). We have shown that COX-2 expression is related to the Type 1 Tyrosine kinase receptor HER2 expression (Boland et al, 2004). From the Kaplan Meier plot for COX-2 and HER2 expression, it is the tumours that express both factors that have a significantly poorer recurrence-free survival advantage over any other group (Figure 2D).

On combining COX-2 and cytoplasmic survivin coexpression (Figure 2E), the cases that express both survivin and COX-2 have the highest 5-year recurrence. Tumours that are both COX-2 and cytoplasmic survivin negative having the greatest disease-free survival advantage; with none of the cases in this series with both receptors negative recurring by 5 years.

In a multivariate analysis, higher cell-proliferation $(P=0.006$; $\operatorname{Exp}(\mathrm{B}) 1.034 ; 95 \%$ Confidence interval (CI) 1.01-1.06), higher nuclear-grade $(P=0.003$; $\operatorname{Exp}(\mathrm{B}) 3.899 ; 95 \% \mathrm{CI} 1.58-9.61)$ and 
Table 2 COX-2 and cytoplasmic survivin co-expression in DCIS

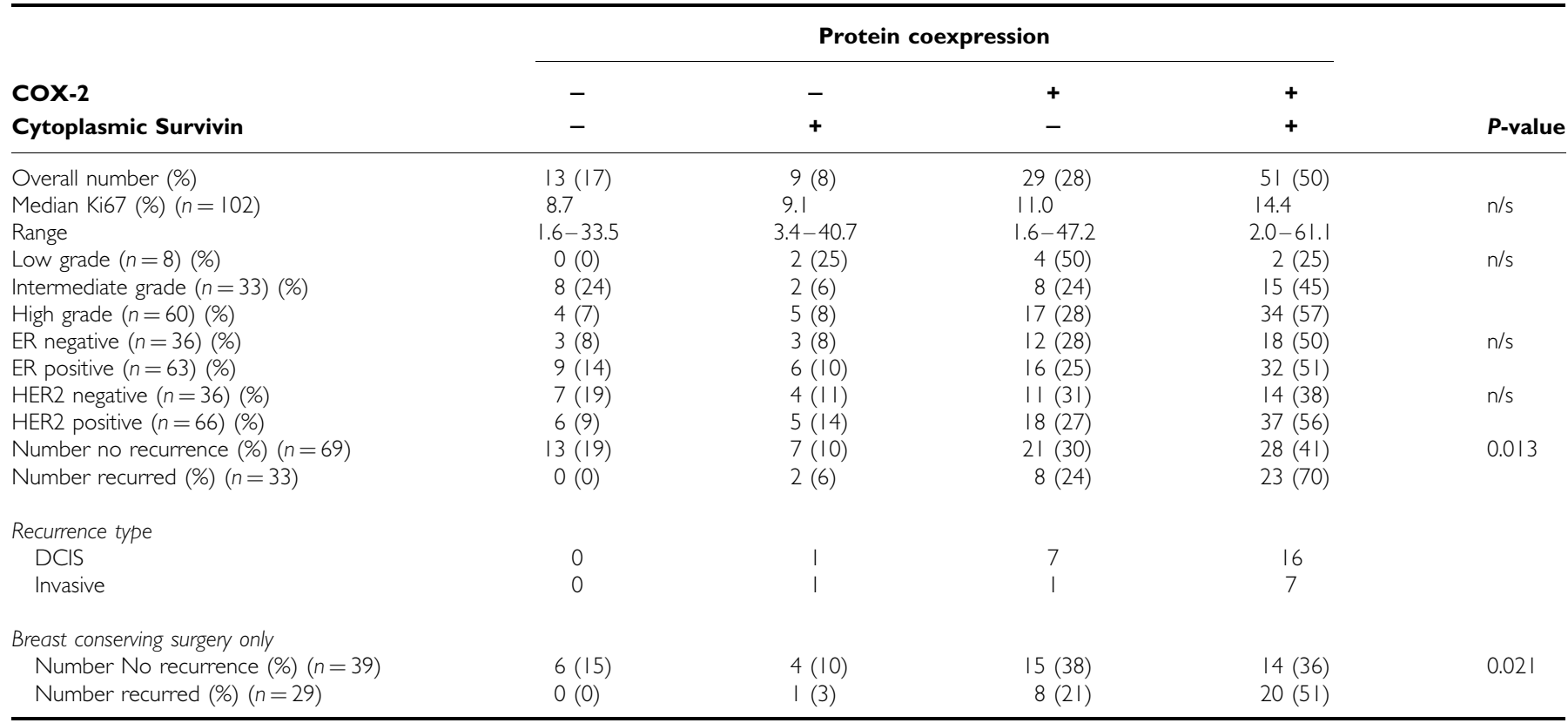

NOTE: $\mathrm{n} / \mathrm{s}=$ not significant; $(n=x \mathrm{x})$ is the total number of patients assessed for each parameter.

higher COX-2 score $(P=0.019 ; \operatorname{Exp}(B) 1.632 ; 95 \%$ CI $1.09-2.46)$ were all independent predictors of increased recurrence of DCIS (also included in the analysis were surgical margin status $(P=0.700)$, HER2 status $(P=0.131)$ and age at diagnosis $(P=0.419))$. The addition of survivin did not aid the model as the two factors correlate in expression frequency.

\section{DISCUSSION}

\section{COX-2 as a predictor of recurrence and therapeutic target}

COX-2 expression in DCIS is a potential predictor of recurrence as well as a therapeutic target. Our own data from breast cancer cell lines, indicates that COX-2 expression inhibits apoptosis, and Celecoxib (a COX-2 inhibitor) reduces xenograft growth by increasing cell death (with no effects on cell proliferation) in a nude mouse model (Barnes et al, 2004).

Here, we have shown that COX-2 expression is associated with early recurrence of DCIS (within 5 years of surgery) and is usually associated with survivin expression. We have shown that the cytoplasmic expression of survivin correlates with COX-2 expression and DCIS recurrence. In this study, staining was prioritised for the evaluation of COX-2 and survivin, insufficient tissue remained on enough blocks after this staining for the additional evaluation of markers of apoptosis. However, COX-2 expression has previously been demonstrated to correlate with low-apoptotic rates (Liu et al, 2001). This supports the hypothesis that the expression of these two factors is linked via the stabilisation of survivin by COX-2 (as found by Krysan et al, 2004) in non-small cell lung cancer), leading to the inhibition of apoptosis. The net effect of which would be to increase the longevity of survivin, thus inhibiting the caspase pathway and increasing cell survival.

\section{Cytoplasmic survivin expression increases recurrence risk}

In IBC, previous studies of survivin protein expression range from 60 (Chu et al, 2004) to $94 \%$ (Ryan et al, 2005). Overall, we were able to demonstrate the presence of survivin in $74 \%$ of IBC and
$73 \%$ of DCIS, with DCIS showing stronger expression levels than invasive cancer; indicating that survivin expression may be an early event in the malignant process.

Survivin, when present in the cell cytoplasm, was associated with increased recurrence of DCIS. We found no correlation between nuclear survivin and recurrence risk. This is a logical finding, as when survivin is in the nucleus it is sub-cellularly distinct from the caspases, which it blocks - which are present and function in the cell cytoplasm. Survivin is, however, a nuclear shuttling protein that exists in three splice variants; wild-type survivin, survivin-2B and survivin delta Ex3. The survivin and survivin $2 \mathrm{~B}$ splice variants are more often found in the cytoplasm, whereas the delta Ex3 is more frequently found in the nucleus (Rodriguez et al, 2002). The antibody we used in this study, detects all three splice variants and further work to characterise the impact of each of these splice variants on recurrence risk needs to be undertaken. Full understanding of whether the differing recurrence/cell signalling effects seen with nuclear $v s$ cytoplasmic survivin are due to the subcellular location, or the differential functions of the splice variants remains to be seen. Antibodies to the separate splice variants are not yet commercially available for use in immunohistochemistry.

\section{Predicting recurrence risk}

The optimum treatment of DCIS is still controversial. In the USA, many cases of DCIS do not receive radiotherapy after breast conserving surgery (Baxter et al, 2004). Prediction of recurrence risk in both DCIS (and invasive cancer), by way of receptor expression profiling may aid decisions about the need for radiotherapy. Panels of robust indicators of recurrence risk (both clinico-pathological and molecular-biological) could potentially be utilised as routine, to direct individual patient treatment. Identifying patients both at high risk of recurrence, who would require close postoperative radiotherapy and also patients with good prognostic factors that may well be able to avoid unnecessary adjuvant radiotherapy or Tamoxifen.

In this study, we have shown for the first time that lack of COX-2 expression is a predictor of a decreased risk of DCIS recurrence, 

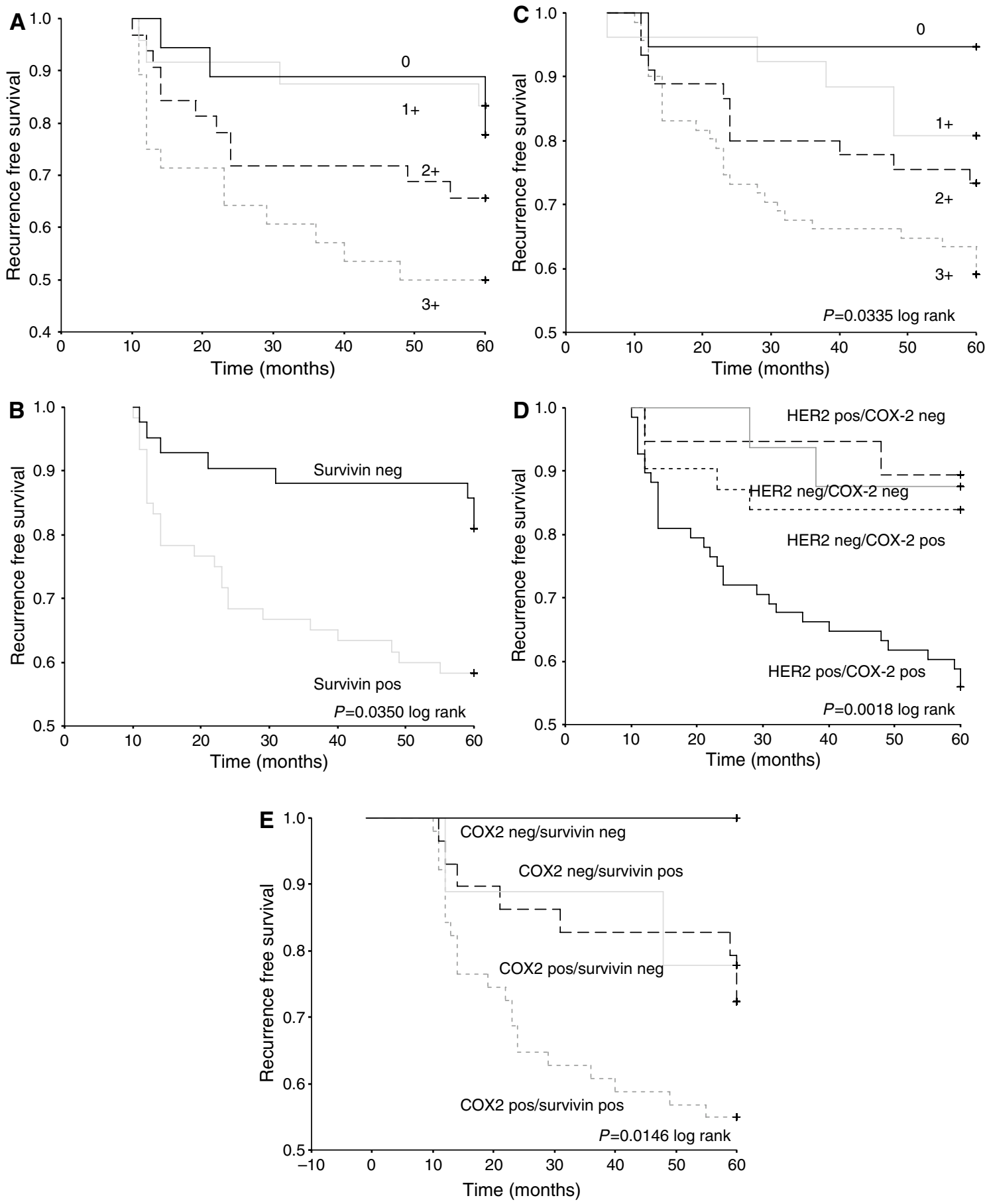

Figure 2 Higher expression of both survivin $(\mathbf{A}$ and $\mathbf{B})$ and COX-2 $(\mathbf{C})$ were associated with increased cumulative 5-year recurrence. DCIS that expressed both COX-2 and HER2 (D) or COX-2 and survivin (E) had the poorest 5-year recurrence-free survival.

that is, DCIS that recurs is rarely COX-2 negative. The potential prediction of early recurrence is enhanced upon determining the coexpression of COX-2 and cytoplasmic survivin, as no cases that expressed neither protein in this series recurred within 5 years. This study was a retrospective look at a large number of DCIS cases. It would be beneficial to look in a prospective series of patients - such as at randomised trial follow up to validate our findings.

In IBC, no cases expressed survivin without COX-2, but in DCIS, survivin was present in nine of 102 cases (9\%), which lacked COX-2. There must, therefore, be alternate pathways through which survivin is stabilised which are independent of COX-2. Alternatively, the ultimate inducer of COX-2 and survivin may be controlled by up stream, potential candidates being the $\mathrm{NF} \kappa \mathrm{B}$ or HER2/Ras pathways. In DCIS, COX-2 expression correlated with HER2, thus understanding the downstream signalling via Ras to COX-2 and survivin splice variants warrants further study.

\section{Inhibiting survivin as a therapeutic target}

Survivin and other IAP's have been reported to be upregulated in several malignancies, including breast cancer (Kawasaki et al, 
1998; Lu et al, 1998; Monzo et al, 1999; Saitoh et al, 1999; Tanaka et al, 2000), compared to normal tissue. Phase I clinical trials using the Isis/Eli-Lilly novel survivin RNA antisense inhibitor (LY21818308) are underway to exploit this expression.

In summary, the presence of cytoplasmic survivin correlates with COX-2 expression and recurrence. High expression of both proteins relates to an increased risk of recurrence of DCIS. Assessing the levels of these proteins may identify patients, who could avoid radiotherapy after breast conserving

\section{REFERENCES}

Ambrosini GA, Adida C, Altieri DC (1997) A novel anti-apoptosis gene, survivin, expressed in cancer and lymphoma. Nat Med 3: 917-921

Barnes NLP, Warnberg F, Farnie G, Jiang W, Anderson E, Bundred NJ (2004) Cyclooxygenase-2 inhibition induces apoptosis via PKB pathway and reduces lymphangiogenesis. Breast Cancer Res Treat 88: S71

Baxter NN, Virnig BA, Durham SB, Tuttle TM (2004) Trends in the treatment of ductal carcinoma in situ of the breast. J Natl Cancer Inst 96: $443-448$

Boland GP, Butt IS, Prasad R, Knox WF, Bundred NJ (2004) COX-2 expression is associated with an aggressive phenotype in ductal carcinoma in situ. Br J Cancer 90: 423-429

Chu JS, Shew JY, Huang CS (2004) Immunohistochemical analysis of survivin expression in primary breast cancers. J Formos Med Assoc 103: $925-931$

Ernster VL, Barclay J, Kerlikowske K, Wilkie H, Ballard-Barbash R (2000) Mortality among women with ductal carcinoma in situ of the breast in the population-based surveillance, epidemiology and end results program. Arch Intern Med 160: 953-958

Fisher ER, Sass R, Fisher B, Wickerham L, Paik SM (1986) Pathologic findings from the national surgical adjuvant breast project (protocol 6) I. Intraductal carcinoma (DCIS). Cancer 57: 197-208

Fortugno P, Wall NR, Giodini A, O'Connor DS, Plescia J, Padgett KM, Tognin S, Marchisio PC, Altieri DC (2002) Survivin exists in immunochemically distinct subcellular pools and is involved in spindle microtubule function. J Cell Sci 115: 575-585

Kawasaki H, Altieri DC, Lu CD, Toyoda M, Tenjo T, Tanigawa N (1998) Inhibition of apoptosis by survivin predicts shorter survival rates in colorectal cancer. Cancer Res 58: 5071-5074

Krysan K, Dalwadi H, Sharma S, Pold M, Dubinett S (2004) Cyclooxygenase 2 -dependent expression of survivin is critical for apoptosis resistance in non-small cell lung cancer. Cancer Res 64: 6359-6362 surgery for DCIS. Future use of inhibitors to both these proteins either alone or in combination offer potential new anticancer interventions.

\section{ACKNOWLEDGEMENTS}

Nicola Barnes was supported by a Royal College of Surgeons of England Research Fellowship.
Liu CH, Chang SH, Narko K, Trifan OC, Wu MT, Smith E, Haudenschild C, Lane TF, Hla T (2001) Overexpression of cyclooxygenase-2 is sufficient to induce tumorigenesis in transgenic mice. J Biol Chem 276: $18563-18569$

Lu CD, Altieri DC, Tanigawa N (1998) Expression of a novel antiapoptosis gene, survivin, correlated with tumor cell apoptosis and p53 accumulation in gastric carcinomas. Cancer Res 58: 1808-1812

Monzo M, Rosell R, Felip E, Astudillo J, Sanchez JJ, Maestre J, Martin C, Font A, Barnadas A, Abad A (1999) A novel anti-apoptosis gene: Reexpression of survivin messenger RNA as a prognosis marker in nonsmall-cell lung cancers. J Clin Oncol 17: 2100-2104

Rodriguez JA, Span SW, Ferreira CG, Kruyt FA, Giaccone G (2002) CRM1mediated nuclear export determines the cytoplasmic localization of the antiapoptotic protein Survivin. Exp Cell Res 275: 44-53

Ryan B, O'Donovan N, Browne B, O'Shea C, Crown J, Hill AD, McDermott E, O'Higgins N, Duffy MJ (2005) Expression of survivin and its splice variants survivin-2B and survivin-DeltaEx3 in breast cancer. Br J Cancer 92: $120-124$

Saitoh Y, Yaginuma Y, Ishikawa M (1999) Analysis of Bcl-2, Bax and Survivin genes in uterine cancer. Int J Oncol 15: 137-141

Schimmer A (2004) Inhibitors of apoptosis proteins:translating basic knowledge into clinical practice. Cancer Res 64: 7183-7190

Smith WL, DeWitt DL, Garavito RM (2000) Cyclooxygenases: structural, cellular, and molecular biology. Annu Rev Biochem 69: 145-182

Tanaka K, Iwamoto S, Gon G, Nohara T, Iwamoto M, Tanigawa N (2000) Expression of survivin and its relationship to loss of apoptosis in breast carcinomas. Clin Cancer Res 6: 127-134

Zhang T, Otevrel T, Gao Z, Ehrlich SM, Fields JZ, Boman BM (2001) Evidence that APC regulates survivin expression: a possible mechanism contributing to the stem cell origin of colon cancer. Cancer Res 61: $8664-8667$ 